\title{
CERTAIN INFINITE ZERO-SUM TWO-PERSON GAMES
}

\author{
A. L. DUlmage and J. E. L. PECK
}

1. Introduction. The theorem of von Neumann, that every finite, zero-sum two-person game has a value, has been extended in various ways to infinite games. In particular Wald (6) has shown that any bounded game in which one player has finitely many pure strategies, has a value. Our interest was aroused by the infinite analogue of the game of "hide and seek" as described by von Neumann (5), which does not appear to fit any of the known cases, unless the matrix is bounded. However, the bounded game is dull since its value is zero. This has led us to give another set of sufficient conditions under which an unbounded infinite game may have a value.

2. Notation and definitions. The game $(I, J, K)$ will consist of two arbitrary sets $I$ and $J$ and a real function $K$ on the product set $I \times J$. If the maximising player chooses $i \in I$ and the minimising player independently chooses $j \in J$, then the former receives the amount $K(i, j)$ from the latter.

Let $\xi=\left\{x_{i} ; i \in I\right\}$ denote a vector with dimension the cardinality of $I$, such that $\sum x_{i}=1$ and all $x_{i} \geqslant 0$, the sum being taken in the sense of Bourbaki (3, Ch. III, §4). The vector $\xi$ will be used as a mixed strategy for the maximising player. Similarly $\eta=\left\{y_{j}\right\}$ will be used as a mixed strategy for the minimising player. We write

$$
K(\xi, \eta)=\sum_{i, j} K(i, j) x_{i} y_{j}
$$

when the expression on the right is summable in the sense of Bourbaki (3). For a particular $\xi$,

$$
\inf _{\eta} K(\xi, \eta)
$$

will denote the infimum over all those $\eta$ for which $K(\xi, \eta)$ exists. We write

$$
\tilde{v}_{I J}=\sup _{\xi} \inf _{\eta} K(\xi, \eta)
$$

and similarly

$$
\bar{v}_{I J}=\inf _{\eta} \sup _{\xi} K(\xi, \eta) .
$$

If the sets $I$ and $J$ are finite then the fundamental theorem states that $\tilde{v}_{I J}=\bar{v}_{I J}$. If $J$ is a finite set and the $K(i, j)$ are bounded then it is known (1) that $\tilde{v}_{I J}=\bar{v}_{I J}$. In the general case, if $K(\xi, \eta)$ exists for all $\xi$ and $\eta$ then

$$
\tilde{v}_{I J} \leqslant \bar{v}_{I J}
$$

Received, October 20, 1955. This work was done while the authors were fellows of the Summer Research Institute of the Canadian Mathematical Congress. 
However, as we may see from an example of Bohnenblust, Karlin and Shapley (2), if there is some $K(\xi, \eta)$ which does not exist, we may have $\tilde{v}_{I J}>\bar{v}_{I J}$.

Games for which (1) holds we call admissible, and we say that any game for which $\tilde{v}_{I J}=\bar{v}_{I J}$ is determinate, or has a value. Any game for which the $K(i, j)$ are bounded below or bounded above is admissible, because all the $K(\xi, \eta)$ exist. It is a simple matter to construct admissible games and in fact determinate games in which, for some $\xi$ and $\eta, K(\xi, \eta)$ does not exist. For any admissible game we have from (1) and the definitions that

$$
\tilde{v}_{I J} \leqslant \bar{v}_{I J} \leqslant \bar{v}_{I n},
$$

for any subset $n$ of $J$.

If $M$ is the collection of finite subsets of $I$ directed by inclusion, then $\lim _{i} f(i)=A$ will mean that for all $\epsilon>0$ there exists an $m \in M$ such that for all $i \notin m,|f(i)-A|<\epsilon$. Similarly $\lim _{m \in M} f(m)=A$ will mean that for all $\epsilon>0$ there exists an $m^{\prime} \in M$ such that for all $m \supseteq m^{\prime},|f(m)-A|<\epsilon$. There are obvious modifications in the case where $A$ is not finite. Similarly $N$ will represent the collection of finite subsets of $J$. We denote the cardinal number of the set $s$ by $|s|$.

\section{A sufficient condition for an admissible game to have a value.}

THEOREM. If the game $(I, J, K)$ is admissible and if for each $j \in J$ there is a real number $L_{j}$ such that

$$
\begin{aligned}
& \inf _{i} K(i, j)=L_{j} \\
& \lim _{i} K(i, j)=L_{j},
\end{aligned}
$$

then the game has a value

$$
v=\lim _{n \in N} \tilde{v}_{I n}
$$$$
v<+\infty
$$

and the maximising player has an optimal strategy.

Proof. For any $n \in N$, the game $(I, n, K)$ is bounded by hypothesis, and so (1) has a value $v_{I n}=\tilde{v}_{I n}=\bar{v}_{I n}$. But if $n \subseteq n^{\prime}$, then $v_{I n} \geqslant v_{I n^{\prime}}$ so that we may write

$$
v=\lim _{n \in N} v_{I n}
$$

and $v<+\infty$. From (2) and (5) we have that

$$
\tilde{v}_{I J} \leqslant \bar{v}_{I J} \leqslant v \text {. }
$$

If $v=-\infty$, then the game has the value $-\infty$ and every strategy for the maximising player is optimal. Thus we need only consider the case where $v$ is finite.

For each $n \in N$ we may choose a strategy $\xi_{n}=\left\{x_{n i}\right\}$ for the maximising player which is $|n|^{-1}$ optimal for the game $(I, n, K)$ in the sense that for all $j \in n$ 


$$
K\left(\xi_{n}, j\right)=\sum_{i} K(i, j) x_{n i} \geqslant v_{\text {In }}-|n|^{-1} .
$$

Since the closed interval $[0,1]$ is compact, it follows from the Tychonoff theorem that the product $\pi$ of $|I|$ of these intervals is compact (4) in the topology of coordinate-wise convergence. Since for every $n \in N, \xi_{n}=\left\{x_{n i}\right\}$ lies in $\pi$, the net $\left\{\xi_{n} ; n \in N\right\}$ has a convergent (4) subnet $\left\{\xi_{n} ; n \in N^{\prime}\right\}$. For each $i$ put

$$
x_{i}{ }^{\prime}=\lim _{n \in N^{\prime}} x_{n i}
$$

Then for every $i \in I$

$$
0 \leqslant x_{i}^{\prime} \leqslant 1, \quad \sum_{i \in I} x_{i}^{\prime} \leqslant 1 .
$$

We now write $\xi^{\prime}=\left\{x_{i}{ }^{\prime}\right\}$ even though $\xi^{\prime}$ may not be a strategy.

Put $L(i, j)=K(i, j)-L_{j}$, then from (3)

$$
L(i, j) \geqslant 0 \text {, }
$$

and from (4),

$$
\lim _{i} L(i, j)=0 .
$$

With the obvious interpretation of $L\left(\xi_{n}, j\right)$ and $L\left(\xi^{\prime}, j\right)$ we shall prove that, for all $j \in J$,

$$
\lim _{n \in N^{\prime}} L\left(\xi_{n}, j\right)=L\left(\xi^{\prime}, j\right) .
$$

In fact, given $\epsilon>0$, choose, by (10), a finite subset $m_{j} \subseteq I$ so that when $i \notin m_{j}, L(i, j)<\frac{1}{3} \epsilon$, and putting

$$
B_{j}=\max _{i} L(i, j),
$$

choose $n^{\prime} \in N^{\prime}$ so that whenever $n$ follows $n^{\prime}$ in $N^{\prime}$ we have for all $i \in m_{j}$ that $\left|x_{n i}-x_{i}{ }^{\prime}\right|<\epsilon\left(3\left|m_{j}\right| B_{j}\right)^{-1}$. Then

$$
\begin{aligned}
& \left|L\left(\xi_{n}, j\right)-L\left(\xi^{\prime}, j\right)\right|=\left|\sum_{i} L(i, j) x_{n i}-\sum_{i} L(i, j) x_{i}{ }^{\prime}\right| \\
& \quad \leqslant \sum_{i \in m_{j}} L(i, j)\left|x_{n i}-x_{i}{ }^{\prime}\right|+\sum_{i \notin m_{j}} L(i, j) x_{n i}+\sum_{i \notin m_{j}} L(i, j) x_{i}{ }^{\prime} \\
& \quad<B_{j} \sum_{i \in m_{j}} \epsilon\left(3\left|m_{j}\right| B_{j}\right)^{-1}+\frac{1}{3} \epsilon \sum_{i} x_{n i}+\frac{1}{3} \epsilon \sum_{i} x_{i}{ }^{\prime} \\
& \quad \leqslant \frac{1}{3} \epsilon+\frac{1}{3} \epsilon+\frac{1}{3} \epsilon=\epsilon .
\end{aligned}
$$

Since for each $j \in n$, we have from (7) that

$$
v_{I n}-|n|^{-1} \leqslant L\left(\xi_{n}, j\right)+L_{j}
$$

it follows from (5) and (11) that for all $j \in J$

$$
v \leqslant L\left(\xi^{\prime}, j\right)+L_{j}
$$

Let $\sum x_{i}{ }^{\prime}=\theta$, then from (8) $0 \leqslant \theta \leqslant 1$ and if $\xi^{*}=\left\{x_{i}{ }^{*}\right\}$ is any mixed strategy, put $\xi=\xi^{\prime}+(1-\theta) \xi^{*}$, that is for all $i, x_{i}=x_{i}{ }^{\prime}+(1-\theta) x_{i}{ }^{*}$. 
From (8) we have that $\sum x_{i}=1$ and that all $x_{i} \geqslant 0$ so that $\xi$ is a mixed strategy. Using (12), (9) and the fact that $x_{i}{ }^{\prime} \leqslant x_{i}$, we have for all $j \in J$ that

$$
v \leqslant \sum_{i} L(i, j) x_{i}{ }^{\prime}+L_{j} \leqslant \sum_{i} L(i, j) x_{i}+L_{j} \sum_{i} x_{i}=\sum_{i} K(i, j) x_{i}=K(\xi, j),
$$

and therefore that

$$
v \leqslant \inf _{j} K(\xi, j)
$$

However, from the definition of $\tilde{v}_{I J}$,

$$
\inf _{j} K(\xi, j) \leqslant \tilde{v}_{I J}
$$

so, using (6),

$$
v \leqslant \inf _{j} K(\xi, j) \leqslant \tilde{v}_{I J} \leqslant \bar{v}_{I J} \leqslant v .
$$

This proves that the game has a value $v$, which by (5) is

$$
\lim _{n \in N} v_{I n}
$$

and that $\xi$ is an optimal strategy for the maximising player.

4. The infinite game of hide and seek. This game is played on a countably infinite matrix $\left(\alpha_{i j}\right)$ where $\alpha_{i j}>0$. The hider chooses a place $(i, j)$ and the seeker chooses either a row $i$ or a column $j$ and if he "finds" the hider, the amount $\alpha_{i j}$ passes from the hider to the seeker. In the finite $n \times n$ case, von Neumann has shown (5) that the value of the game is $S_{n}^{-1}$, where, if $P_{n}$ is some permutation of the integers $i=1, \ldots, n$,

$$
S_{n}=\max _{P_{n}} \sum_{i=1}^{n}\left(\alpha_{i i^{P_{n}}}\right)^{-1} \text {. }
$$

We observe, in both the finite and the infinite case, that corresponding to every pure strategy of the hider, the seeker has only two pure strategies in which the pay-off is positive and in all other cases it is zero. If this game is considered in the normal form $(I, J, K)$, this means that, for each $j$, every $K(i, j)$ is zero except for two which are positive. The game is clearly admissible, since the $K(i, j)$ are bounded below by zero. The conditions of our theorem are easily satisfied with $L_{j}=0$ for all $j$ and the infinite game therefore has the value

$$
v=\lim _{n \rightarrow \infty} v_{n}
$$

where $v_{n}$ is the value of the game $\left(\infty, n^{2}, K\right)$. However the game $\left(\infty, n^{2}, K\right)$ is clearly equivalent to the game $\left(2 n, n^{2}, K\right)$, whose value is $S_{n}^{-1}$, because if the hider is restricted to a square, the seeker would not seek outside it. Thus

$$
v=\lim _{n \rightarrow \infty} v_{n}=\lim _{n \rightarrow \infty} S_{n}^{-1}
$$


If we let $P$ be any permutation of the set of positive integers onto itself and if

$$
S=\sup _{P} \sum_{i=1}^{\infty}\left(\alpha_{i i^{P}}\right)^{-1}
$$

then it is easily shown that

$$
\lim _{n \rightarrow \infty} S_{n}=S
$$

and therefore that $v=S^{-1}$. In fact, if $S<\infty$, then we can choose $P$ so that

$$
\sum_{i=1}^{\infty}\left(\alpha_{i i^{P}}\right)^{-1}>S-\frac{1}{2} \epsilon
$$

and then choose $n$ so that

$$
\sum_{i=1}^{n}\left(\alpha_{i i}\right)^{-1}>S-\epsilon
$$

If

$$
m=\max _{1 \leqslant i \leqslant n} i^{P}
$$

then we have

$$
S \geqslant S_{m} \geqslant \sum_{i=1}^{n}\left(\alpha_{i i^{P}}\right)^{-1}>S-\epsilon .
$$

The case $S=\infty$ is similar. This justifies our statement in $\$ 1$ that the value of the bounded game is zero, since in that case $S=\infty$.

There exist unbounded hide and seek games in which the value is not zero, for example if $\alpha_{i j}=2^{\max (i, j)}$, the value of the game is 1 .

There is the same connection between the game of hide and seek and the optimal assignment problem, as in the finite case, but with obvious modifications.

More general games of hide and seek can be considered as played on a $t$-dimensional array $\left(\alpha_{i_{1}}, \ldots, i_{t}\right)$ where the hider chooses a place $\left(i_{1}, \ldots, i_{t}\right)$ and the seeker chooses some $r$ subscripts, $r \leqslant t$. Our theorem shows that such infinite games have a value.

\section{REFERENCES}

1. D. Blackwell and M. A. Girshick, Theory of Games and Statistical Decisions (Wiley, 1954).

2. H. F. Bohnenblust, S. Karlin and L. S. Shapley, Solutions of discrete games, Ann. Math. Studies, 24 (1950), 51-72.

3. N. Bourbaki, Topologie générale (Paris, Hermann).

4. J. L. Kelley, Convergence in topology, Duke Math. J., 17 (1950), 277-283.

5. J. von Neumann, A certain zero-sum two-person game equivalent to the optimal assignment problem, Ann. Math. Studies, 28 (1953), 5-12.

6. A. Wald, Generalization of a theorem by v. Neumann concerning zero sum two person games, Ann. Math., 46 (1945), 281-286. 